\title{
Control of Multivariable Hammerstein Systems by Using Feedforward Passivation
}

\author{
Steven W. Su${ }^{\dagger}$, Jie Bao ${ }^{\dagger}$ and Peter L. Lee \\ ${ }^{\dagger}$ School of Chemical Engineering \& Industrial Chemistry \\ The University of New South Wales, \\ UNSW, Sydney, NSW 2052, Australia \\ ${ }^{\ddagger}$ Division of Engineering, Science and Computing \\ Curtin University of Technology \\ GPO Box U1987, Perth, WA 6845, Australia
}

\begin{abstract}
This paper presents a new control method for processes which can be described by Hammerstein models. The control design is based on the concept of passive systems. The proposed method is based on feedforward passivation and thus can be applied to non-minimum phase processes and/or processes of high relative degree. A synthesis technique for marginally stable positive real systems are developed to achieve offset free control. The new control design can be easily implemented by solving a set of linear matrix inequalities. The proposed approach is illustrated using the example of an acid-base $\mathrm{pH}$ control problem.
\end{abstract}

Keywords: Hammerstein model, integral control, passive systems, linear matrix inequality 


\section{Introduction}

A linear system with an input nonlinearity is known as a Hammerstein system. The time invariant state space representation of a multivariable nonlinear continuous-time Hammerstein system can be described by:

$$
P:\left\{\begin{array}{l}
\dot{x}=A x+B \cdot N L(u) \\
y=C x+D \cdot N L(u) .
\end{array}\right.
$$

Hammerstein models may account for nonlinear effects encountered in many chemical processes ${ }^{[1]}$. For example, the nonlinear behavior of distillation columns ${ }^{[2]}, \mathrm{pH}$ neutralization processes ${ }^{[3]}$, heat exchangers ${ }^{[4,5]}$, furnaces and reactors ${ }^{[6]}$ can be effectively modelled by a nonlinear static element cascaded by a linear dynamic element. Therefore, a control method based on a Hammerstein model is of practical importance in the process industries.

Most existing control methods for Hammerstein systems are based on the direct inversion of the static nonlinearity (e.g., the nonlinear control law presented in [7] and [8]). However, inverting multivariable nonlinear functions is often very difficult. While analytic solutions are usually unavailable, direct inversion is normally implemented numerically, which requires intensive on-line computation. In addition, inversion of static nonlinearities could be very sensitive to the uncertainties in the nonlinear functions. Theoretically, inversion methods cannot be used to control processes that exhibit input multiplicity.

Methods based on nonlinear model predictive control (nonlinear MPC) ${ }^{[8,9,6,10]}$ have also been developed for control of Hammerstein systems. The main shortcoming of this approach is that it requires non-convex on-line dynamic optimization ${ }^{[11]}$. The reliability and efficiency of the existing methods still needs improvement ${ }^{[12]}$.

Based on feedforward passivation, this paper presents a new control strategy for Hammerstein systems, which guarantees closed loop stability and ensures offset free tracking. Offset free control is desirable in chemical process control despite model uncertainties, unknown disturbances and slow variations of process dynamics ${ }^{[13]}$. The basic idea is to modify the process model using a feedforward (parallel compensation) such that the modified Hammerstein system is easy to control. Parallel compensation has been used in Internal Model Control ${ }^{[14,15]}$ and Dead Time Compensation ${ }^{[16]}$ to ensure closed-loop stability. It was also applied to cancel the effect of the destabilizing nonlinear elements in control of nonlinear systems $([17])$. Parallel compensation, while useful in stability 
design, needs to be implemented in conjunction with other techniques to achieve desirable control performance. To achieve offset free control, integral action has to be used in the controller. Such controllers are better constructed based on the Passivity Theorem, which can deal with systems with unlimited gain explicitly. Therefore, a dynamic feedforward system is implemented to render the process dynamic passive (similar to [18], [19] and [20]). A systematic approach is developed in this work to construct feedforward passivation and the overall control system such that zero steady state error and fast tracking responses can be achieved. The feedforward "passifier" is constructed by solving a set of linear matrix inequalities (LMI). This approach does not require the linear dynamics of the Hammerstein system to be positive real, as required in [21] and [22]. Furthermore, this design method can be applied to processes of relative degree greater than one and/or non-minimum phase, unlike the approaches based on feedback passivation ${ }^{[23]}$.

In the next section, the passivity-based stability condition is reviewed briefly. The new control approach is described in Section 3. This includes the sufficient condition for offset free control using feedforward passivation for nonlinear MIMO processes, the details of control design and the numerical design tool using Semi-Definite Programming technique. In Section 4, the control of an acid-base neutralization process is presented to show the effectiveness of the proposed method.

Discussion and conclusions are provided in Section 5.

\section{Preliminaries}

The cornerstone of the proposed control method is the concept of passive systems and the Passivity Theorem $[24,25,19]$.

Consider a nonlinear system $H$ :

$$
\begin{cases}\dot{x}=f(x, u) & x \in \mathcal{X} \subseteq \mathcal{R}^{n} \\ y=h(x, u), & u \in \mathcal{U} \subseteq \mathcal{R}^{m}, \quad y \in y \subseteq \mathcal{R}^{m}\end{cases}
$$

and assume that the state $x(t)$, as a function of time, is uniquely determined by its initial value $x(0)$ and the input function $u(t)$. Suppose that the above system has an equilibrium at the origin, that is, $f(0,0)=0$, and $h(0,0)=0$.

Definition $1{ }^{[19]}$ Assume that associated with the system $H$ is a function $w: \mathcal{R}^{m} \times \mathcal{R}^{m} \mapsto \mathcal{R}$, called the supply rate, which is locally integrable for every $u \in \mathcal{U}$. Let $X$ be a connected subset of $\mathcal{R}^{n}$ 
containing the origin. If there exists a function $S: \mathcal{X} \mapsto \mathcal{R}^{+}$(denote $\mathcal{R}^{+}=[0, \infty)$ ), $S(0)=0$, such that for all $x \in \mathcal{X}$ :

$$
S(x(T))-S(x(0)) \leq \int_{0}^{T} w(u(t), y(t)) d t
$$

for all $u \in \mathcal{U}$ and all $T \geq 0$ such that $x(t) \in \mathcal{X}$ for all $t \in[0, T]$, then we say that the system $H$ is dissipative in $X$ with the supply rate $w(u, y)$. The function $S(x)$ is then called a storage function.

Definition 2 System $H$ is said to be passive if it is dissipative with supply rate $w(u, y)=u^{T} y$.

For linear systems, the definition of passive systems is reduced to:

Definition $3{ }^{[26]}$ A linear time-invariant system, $G(s)=C(s I-A)^{-1} B+D,(G(s)$ is a $m \times m$ transfer matrix), is passive if and only if $G(s)$ is Positive Real (PR), or equivalently,

i) $G(s)$ is analytic in $\operatorname{Re}(s)>0$

ii) $G(j \omega)+G^{*}(j \omega) \geq 0$ for all frequency $\omega$ such that $j \omega$ is not a pole of $G(s)$. If there are poles $p_{1}, p_{2}, \cdots, p_{m}$ of $G(s)$ on the imaginary axis, they are nonrepeated and the residue matrix at the poles $\lim _{s \rightarrow p_{i}}\left(s-p_{i}\right) G(s)(i=1,2, \cdots, m)$ is Hermitian and positive semi-definite.

System $G(s)$ is said to be strictly passive or Strictly Positive Real (SPR) if [27]

i) $G(s)$ is analytic in $\operatorname{Re}(s) \geq 0$

ii) $G(j \omega)+G^{*}(j \omega)>0$ for $\omega \in(-\infty,+\infty)$.

For nonlinear systems, the concept of strict passivity can be represented by Output Feedback Passivity $(O F P)$ and Input Feedforward Passivity $(I F P)$ :

Definition $4^{[19]}$ System $H$ is said to have:

- Output Feedback Passivity $(O F P(\rho))$ if it is dissipative with respect to $w(u, y)=u^{T} y-\rho y^{T} y$ for some $\rho \in \mathcal{R}$.

- Input Feedforward Passivity $(\operatorname{IFP}(\nu))$ if it is dissipative with respect to $w(u, y)=u^{T} y-\nu u^{T} u$ for some $\nu \in \mathcal{R}$.

The excess and shortage of passivity are evaluated by the notation $\operatorname{OFP}(\rho)$ and $\operatorname{IFP}(\nu)$, where a positive sign of $\rho$ or $\nu$ implies that the system has an excess of passivity. The $O F P(\rho)$ systems (or IFP $(\nu)$ systems) remain passive under static positive feedback $\rho I$ (or negative static feedforward $\nu I)$. 
Passivity is an input-output property. In order to use the passivity condition to determine closed-loop stability of interconnected systems, introduction of the following definition is necessary:

Definition $5{ }^{[19]}$ Consider the system $H$ with zero input, that is $\dot{x}=f(x, 0), y=h(x, 0)$, and let $Z \in \mathcal{R}^{n}$ be its largest positively invariant set contained in $\left\{x \in \mathcal{R}^{n} \mid y=h(x, 0)=0\right\}$. System $H$ is zero-state detectable (ZSD) if $x=0$ is asymptotically stable conditionally to $Z$.

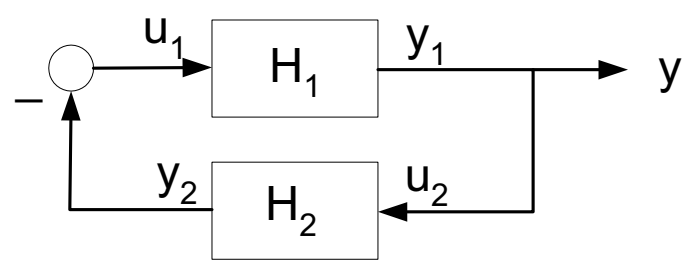

Figure 1: Feedback interconnections of two systems

On the basis of the concept of passive systems, the stability of two interconnected nonlinear systems can be determined by the following passivity theorem:

Theorem $6{ }^{[19]}$ Assume that the systems $H_{1}$ and $H_{2}$ are dissipative with the supply rates:

$$
w_{i}\left(u_{i}, y_{i}\right)=u_{i}^{T} y_{i}^{T}-\rho_{i} y_{i}^{T} y_{i}-\nu_{i} u_{i}^{T} u_{i}, i=1,2 .
$$

Furthermore assume that they are ZSD. Then the equilibrium $\left(x_{1}, x_{2}\right)=(0,0)$ of the feedback interconnection in Figure 1 is asymptotically stable, if $\nu_{1}+\rho_{2}>0$ and $\nu_{2}+\rho_{1}>0$.

The supply rates (4) in Theorem 6 are generalizations of the supply rates for OFP and IFP systems (see Definition 4). Theorem 6 implies the well known result that a feedback system comprised of a passive system and a strictly passive system is asymptotically stable. Furthermore, it shows that a shortage of passivity of one system can be compensated by the excess of passivity of the other, and this ensures the overall closed loop stability, provided that state detectability requirement is satisfied.

For a static nonlinearity $N L(\cdot)$, as its state space is void, the only choice for its storage function is $S \equiv 0$. Thus, the dissipativity of $N L(\cdot)$ with the supply rate in equation (4) can be determined by the inequality $N L^{T}(u) u-\rho N L^{T}(u) N L(u)-\nu u^{T} u \geq 0$. 


\section{Controller Design}

\subsection{Control Framework}

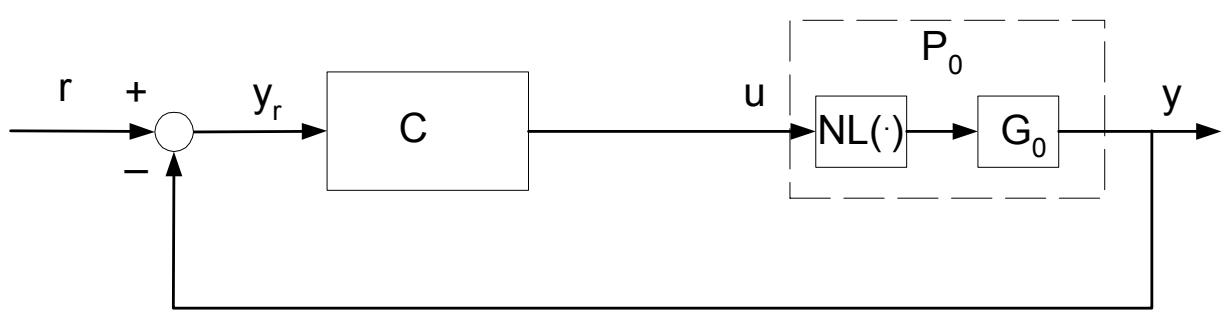

Figure 2: A nonlinear offset free control problem

The control problem can be described as follows. Consider the feedback system of a stable process $P_{0}$, described by a Hammerstein model (a nonlinearity $N L(\cdot)$ cascaded by a linear dynamic element $\left.G_{0}(s)\right)$ and a controller $C$, as shown in Figure 2. It is assumed that the number of inputs is equal to that of the outputs. Signal $y_{r}$ is the reference tracking error which is equal to $r-y$. Signal $u$ is the input to the plant. A controller $C$ is designed to guarantee zero steady state tracking error for a constant reference input $r$.

It is well known that integral control is essential to attaining offset free tracking of both linear and nonlinear systems ${ }^{[28]}$. Therefore, a controller with integral action, $C=C^{\prime} / s$, should be used. According to Theorem 6, if the nonlinearity $N L(\cdot)$ satisfies the inequality $N L^{T}(u) u-$ $\rho N L^{T}(u) N L(u)-\nu u^{T} u \geq 0$, then the controller can be designed such that the linear part of the open-loop system (including the linear parts of the controller and the process) is $\operatorname{IFP}(\tilde{\nu})$, with $\rho+\tilde{\nu}>0$ and $\nu>0$, to guarantee asymptotic stability of the closed-loop system (provided both the process and the controller are ZSD). This can be achieved by implementing a feedforward system $\left(G_{f f}(s)\right)$ as shown in Figure 3. As the output of the static nonlinear part of the process is not obtainable, the actual feedforward $P_{f f}$ includes the model of the static nonlinearity $N L(\cdot)$. The final controller is $C^{\prime} / s$ with the negative feedback of $P_{f f}$. The proposed approach, which involves passivation of only the linear dynamics of the open-loop system, allows systematic controller design. This approach can be applied directly to Wiener systems as the open-loop system of a controller and a Wiener process can also be regrouped into a linear part and a nonlinear part. 


\subsection{Passivity-based Controller Design}

The following considers conditions under which the feedforward passivation design can achieve offset free tracking. The proposed control design includes the construction of the dynamic feedforward $\left(P_{f f}\right)$ element and the linear controller with integral action $\left(C^{\prime} / s\right)$.

In order to clarify our discussions, we define two notations first.

Definition 7 We call a diagonal square matrix $\frac{K}{s}$ (assume the dimension of this matrix is $m$ ) with diagonal elements as $k_{i} / s\left(k_{i}>0\right), i \in\{1,2, \cdots, m\}$ an integration matrix.

Definition 8 Consider an input output stable nonlinear system $\mathcal{P}$, which is described by equation (2). If for any constant input $u_{c} \in \mathcal{U}$, the steady state output $y_{c}$ of the system $\mathcal{P}$ is equal to zero, i.e.

$$
y_{c}=\lim _{t \rightarrow \infty} \mathcal{P}\left(u_{c}\right)=0,
$$

then, system $\mathcal{P}$ is said to have zeros at steady state.

The following theorem shows that if the feedforward subsystem has zeros at steady state, then the integral action in the controller can be preserved to achieve offset free tracking.

Theorem 9 Consider the system depicted in Figure 4 (the reference input $r=0$ ). Suppose that the plant $P$ (which is the sum of two subsystems $P_{0}$ and $P_{f f}$ ) can be described by equation (2). Further assume the two functions $f: \mathcal{R}^{n} \times \mathcal{R}^{m} \mapsto \mathcal{R}^{n}$ and $g: \mathcal{R}^{n} \times \mathcal{R}^{m} \mapsto \mathcal{R}^{m}$ in equation (2) are unbiased in the sense that:

$$
\left\{\begin{array}{l}
f(0,0)=0 \\
g(0,0)=0,
\end{array}\right.
$$

which ensures that $x=0$ is an equilibrium of the unforced system:

$$
\dot{x}=f(x, 0) .
$$

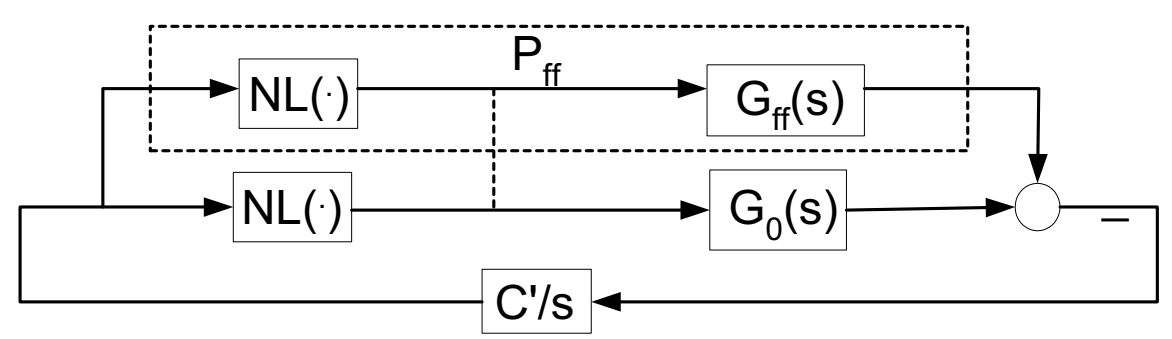

Figure 3: Offset free control for Hammerstein model by using feedforward passivity design 


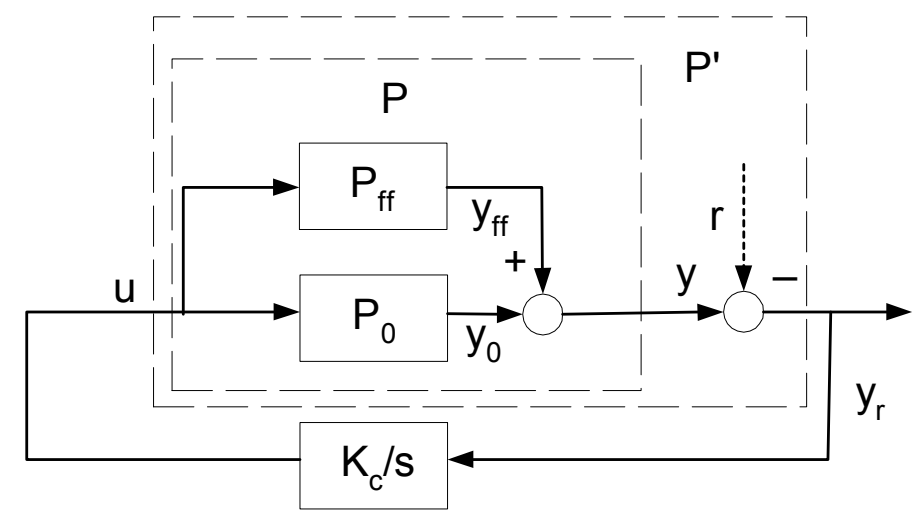

Figure 4: Offset free tracking by using feedforward passivation

Assume that 0 is a Globally Asymptotically Stable equilibrium of plant $P_{0}$, and $(0,0)$ is a Globally Asymptotically Stable equilibrium of the unforced closed loop $\left(P, \frac{K_{c}}{s}\right)$. Further assume that the subsystem $P_{f f}$ has zeros at steady state (see Definition 8), i.e.

$$
y_{c}=\lim _{t \rightarrow \infty} P_{f f}\left(u_{c}\right)=\lim _{t \rightarrow \infty} y_{f f}(t)=0 .
$$

Then the output of the true plant $P_{0}$ will asymptotically go to zero. That is:

$$
\lim _{t \rightarrow \infty} y_{0}(t)=0
$$

Proof The proof is in Appendix A.

Any equilibrium $x_{e}$ under investigation can be translated to the origin by redefining the state $x$ as $x-x_{e}{ }^{[19]}$. For simplicity, in most of the following exposition we will assume that such a translation has already been performed. Thus, for most parts of this paper, the equilibrium under investigation will be $x_{e}=0$. When we need to emphasize a non-zero equilibrium, we will use $x=x_{e}$ as the equilibrium point instead of $x=0$.

In Figure 4, if there is a nonzero constant reference input $r$, the original plant $P_{0}$ and reference input $r$ is equivalent to a new plant $P^{\prime}$ with an equilibrium point $\left(x_{e}\right)$, where $g\left(x_{e}, u_{r}\right)=r$. The sufficient condition for stability in this situation is that the condition of Theorem 9 is satisfied for the new equilibrium point.

For a Hammerstein system (see Figure 3), the subsystem $P_{f f}$ should be designed as the serial connection of the nonlinearity $N L(\cdot)$ and a linear system which has a blocking zero at the origin, so that offset free control can be achieved.

Since the linear block of the closed loop system is $\frac{1}{s} C^{\prime} G_{0}(s)$, the passivity of a linear system with integral action (the passivity of a marginally stable system ${ }^{[29]}$ ) needs to be investigated. 
Lemma 10 Assume that $\{A, B, C, D\}$ is a minimal realization of an $m \times m$ rational stable matrix $G(s)$. Then, the marginally stable matrix $\frac{1}{s} G(s)$ can be decomposed as:

$$
\frac{1}{s} G(s)=\frac{1}{s} G(0)+\hat{G}(s)
$$

where $\hat{G}(s)=C[s I-A]^{-1} A^{-1} B$ is stable.

Proof Consider that (equation (11) can be checked by left multiplication by the matrix $s I-A$ for both sides)

$$
(s I-A)^{-1}=-A^{-1}+s[s I-A]^{-1} A^{-1},
$$

we have:

$$
\begin{aligned}
\frac{1}{s} G(0)+\hat{G}(s) & =-\frac{C A^{-1} B-D}{s}+C[s I-A]^{-1} A^{-1} B \\
& =\frac{1}{s} C\left\{-A^{-1}+s[s I-A]^{-1} A^{-1}\right\} B+\frac{D}{s} \\
& =\frac{1}{s}\left\{C[s I-A]^{-1} B+D\right\} \\
& =\frac{1}{s} G(s) .
\end{aligned}
$$

Theorem 11 Assume $G(s)$ and $Q(s)$ are two $m \times m$ rational stable matrices. Suppose that $\{A, B, C, D\}$ is a minimal realization of $G(s)$, then the marginally stable matrix $\frac{1}{s} G(s)+Q(s)$ is passive if and only if:

i) $G(0)$ is a symmetric and nonnegative definite matrix, i.e. $G(0) \geq 0$.

ii) The matrix $\hat{G}(s)+Q(s)$ is passive. Where $\hat{G}(s)=C[s I-A]^{-1} A^{-1} B$.

Proof ("if" "£")

According to Lemma 10, we have:

$$
\frac{1}{s} G(s)+Q(s)=\frac{1}{s} G(0)+\hat{G}(s)+Q(s)
$$

with $\hat{G}(s)$ being stable. Because $G(0)$ is a symmetric and nonnegative definite matrix as stated in Condition i), it can be seen that:

$$
\lim _{s \rightarrow 0} s \frac{G(0)}{s}=G(0)
$$

is symmetric and nonnegative definite. Consider that:

$$
\frac{G(0)}{j \omega}+\frac{G^{T}(0)}{-j \omega}=0, \omega \in(-\infty, 0) \cup(0, \infty),
$$


given that $G(0)$ is symmetric, we conclude that the transfer function $\frac{G(0)}{s}$ is passive.

From Condition ii) and equation (13), we can draw the conclusion that $\frac{G(s)}{s}+Q(s)$ is passive.

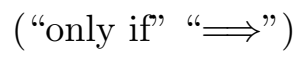

If $\frac{G(s)}{s}+Q(s)$ is passive, then according to Definition 3, we conclude that:

$$
\lim _{s \rightarrow 0} s\left[\frac{G(s)}{s}+Q(s)\right]=G(0)
$$

is symmetric and nonnegative definite.

Furthermore, if $\frac{G(s)}{s}+Q(s)$ is passive, then,

$$
\frac{G(j \omega)}{j \omega}+\frac{G^{T}(-j \omega)}{-j \omega}+Q(j \omega)+Q^{T}(-j \omega) \geq 0, \omega \in(-\infty, 0) \cup(0, \infty) .
$$

Based on Lemma 10 and equation (17), we have:

$$
\frac{G(0)}{j \omega}-\frac{G^{T}(0)}{j \omega}+\hat{G}(j \omega)+\hat{G}^{T}(-j \omega)+Q(j \omega)+Q^{T}(-j \omega) \geq 0, \omega \in(-\infty, 0) \cup(0, \infty) .
$$

That is the rational stable matrix $\hat{G}(s)+Q(s)$ is passive.

Based on Lemma 10 and Theorem 11, an integral control approach can be proposed which is embodied in Theorem 12. A marginally stable LTI system, while theoretically can be passified by a feedforward subsystem, leads to positive semi-definite conditions which cannot be solved numerically. The following theorem decomposes the linear part of the open-loop system into the marginally stable and asymptotically stable components. It specifies the conditions that the asymptotically stable component has to meet such that the closed-loop system is stable. Such conditions can be translated into strictly positive definite matrix inequality constraints.

Theorem 12 Consider the controlled Hammerstein system depicted in Figure 3. Assume the integral controller $\left[\frac{C^{\prime}(s)}{s}\right]_{m \times m}$ has the following form:

$$
\frac{C^{\prime}(s)}{s}=C_{H}+\frac{C_{L}(s)}{s} .
$$



Figure 5: Controller structure 
Where $C_{H}$ is a real matrix and $C_{L}(s)$ is a stable proper transfer function. Further assume the linear transfer function $G_{0}(s)$ is stable and $G_{0}(0)$ is nonsingular. The feedforward system $G_{f f}(s)=$ $s \hat{G}_{f f}(s)\left(\hat{G}_{f f}(s)\right.$ is a strictly proper stable transfer function). Suppose that the nonlinearity $N L(\cdot)$ satisfies:

$$
N L^{T}(u) u \geq \nu u^{T} u+\rho N L^{T}(u) N L(u), \quad \forall u \in \mathcal{U}
$$

with $\nu>0$. Then, the overall closed loop is GAS if one can find a controller $\frac{C^{\prime}(s)}{s}$, a real matrix $\hat{D}_{f f}$ and a strictly proper linear system $\hat{G}_{f f}(s)$ such that:

i) $C^{\prime}(0) G(0)$ is a symmetric and nonnegative definite matrix, i.e. $C^{\prime}(0) G(0) \geq 0$,

ii) $\left(C_{H}+\hat{C}_{L}(s)\right)\left(G_{f f}(s)+G_{0}(s)\right)+C^{\prime}(0)\left(\hat{G}_{f f}(s)+\hat{G}_{0}(s)\right)+\hat{D}_{f f}$ is Positive Real (where $\hat{C}_{L}(s)$ and $\hat{G}_{0}(s)$ are derived from $\frac{1}{s} C_{L}(s)$ and $\frac{1}{s} G_{0}(s)$ respectively as shown in equation (10) of Lemma 10),

iii) $\hat{D}_{f f}+\hat{D}_{f f}^{T}<2 k_{D} I$ and $k_{D}<\rho$.

In addition, if:

iv) $C^{\prime}(s) \hat{G}_{f f}(s)+\hat{D}_{f f}$ is Positive Real,

is also satisfied, then the Global Stability (not GAS) of the controller (see Figure 5) is also guaranteed.

Proof Firstly, we prove the overall closed loop is GAS under Conditions i), ii) and iii). Denote $\bar{G}(s)=\frac{C^{\prime}(s)}{s}\left[G_{0}(s)+s \hat{G}_{f f}(s)\right]$. Because both $G_{0}(0)$ and $C^{\prime}(0)$ are nonsingular, $\bar{G}(s)$ have no transmission zero at the origin. This implies that $\bar{G}(s)$ is ZSD. According to Theorem 6 (regarding the nonlinearity $N L(\cdot)$ as $H_{1}$ in Theorem 6 , and $\bar{G}(s)$ as $\left.H_{2}\right)$, we only need to verify that $\bar{G}(s)$ is $\operatorname{IFP}\left(-k_{D}\right)$.

Because

$$
\bar{G}(s)+\hat{D}_{f f}=\frac{C^{\prime}(0) G_{0}(0)}{s}+\left(C_{H}+\hat{C}_{L}(s)\right)\left(G_{f f}(s)+G_{0}(s)\right)+C^{\prime}(0)\left(\hat{G}_{f f}(s)+\hat{G}_{0}(s)\right)+\hat{D}_{f f}
$$

and $C^{\prime}(0) G(0)$ is a symmetric and nonnegative definite matrix (Condition i)), according to Theorem $11, \bar{G}(s)+\hat{D}_{f f}$ is Positive Real if and only if Condition ii) is satisfied.

Because $\bar{G}(s)+\hat{D}_{f f}$ is Positive Real ( under Condition i) ) and $\hat{D}_{f f}+\hat{D}_{f f}^{T}<2 k_{D} I$ ( Condition ii) ), it is concluded that $\bar{G}(s)+k_{D} I$ is Positive Real, that is, $\bar{G}(s)$ is $\operatorname{IFP}\left(-k_{D}\right)$.

The GAS of observable states of the offset free controller can be directly proved based on the Passivity Theorem. The unobservable states (the states of integrators) are GS (not GAS). Therefore, the overall offset free controller is GS. 
The condition described by inequality (19) requires the nonlinearity to be dissipative with respect to the supply rate of $N L^{T}(u) u-\nu u^{T} u-\rho N L^{T}(u) N L(u)$, where $\nu>0$ and could be arbitrarily small. This condition requires the static nonlinearity to be passive and have a finite gain in the operating region. While this condition cannot be easily met by processes globally (for any input region), it can often be satisfied within certain operating regimes.

Based on the Positive Real Lemma, $\hat{D}_{f f}$ and $\hat{G}_{f f}(s)$ can be found by solving the following matrix inequality problem with the constraints given in Theorem 12. Suppose that $\left\{\hat{A}_{c}, \hat{B}_{c}, \hat{C}_{c}\right\}$, $\{\hat{A}, \hat{B}, \hat{C}\}$ and $\left\{\hat{A}_{f f}, \hat{B}_{f f}, \hat{C}_{f f}\right\}$ are minimal realizations of the strictly proper LTI systems $\hat{C}_{L}(s)$, $\hat{G}_{0}(s)$ and $\hat{G}_{f f}(s)$ respectively. Further assume $\hat{A}_{f f}=\hat{A}$ and $\hat{B}_{f f}=\hat{B}$. As passivity is a phase related property, this assumption, while significantly simplifying the control approach, does not lead to a conservative design. A state space realization of $C^{\prime}(s) \hat{G}_{f f}(s)+\hat{D}_{f f}$ is:

$$
\begin{gathered}
\mathcal{A}_{f f}=\left[\begin{array}{cc}
\hat{A} & 0 \\
\hat{B}_{c} \hat{C}_{f f} & \hat{A}_{c}
\end{array}\right], \quad \mathcal{B}_{f f}=\left[\begin{array}{c}
\hat{B} \\
0
\end{array}\right], \\
\mathcal{C}_{f f}=\left[C_{L}(0) \hat{C}_{f f}+C_{H} \hat{C}_{f f} \hat{A}+\hat{C}_{c} \hat{B}_{c} \hat{C}_{f f} \quad \hat{C}_{c} \hat{A}_{c}\right], \quad \mathcal{D}_{f f}=C_{H} \hat{C}_{f f} \hat{B}+\hat{D}_{f f} .
\end{gathered}
$$

A state space realization of $\left(C_{H}+\hat{C}_{L}(s)\right)\left(G_{f f}(s)+G_{0}(s)\right)+C^{\prime}(0)\left(\hat{G}_{f f}(s)+\hat{G}_{0}(s)\right)+\hat{D}_{f f}$ is:

$$
\begin{gathered}
\mathcal{A}=\left[\begin{array}{cc}
\hat{A} & 0 \\
\hat{B}_{c}\left(\hat{C}_{f f} \hat{A}+\hat{C} \hat{A}\right) & \hat{A}_{c}
\end{array}\right], \quad \mathcal{B}=\left[\begin{array}{c}
\hat{B} \\
\hat{B}_{c}\left[\left(\hat{C}_{f f}+\hat{C}\right) \hat{B}+G_{0}(0)\right]
\end{array}\right], \\
\mathcal{C}=\left[C_{H}\left(\hat{C}_{f f} \hat{A}+\hat{C} \hat{A}\right)+C_{L}(0)\left(\hat{C}_{f f}+\hat{C}\right) \quad \hat{C}_{c}\right], \quad \mathcal{D}=C_{H}\left[\left(\hat{C}_{f f}+\hat{C}\right) \hat{B}+G_{0}(0)\right]+\hat{D}_{f f} .
\end{gathered}
$$

The following minimization problem is established to construct $\hat{D}_{f f}$ and $\hat{G}_{f f}(s)$ based on Positive Real Lemma.

Problem $13 \min _{\hat{C}_{f f}, \hat{D}_{f f}, P_{1}, P_{2}} \alpha$

Subject to:

$$
\begin{gathered}
{\left[\begin{array}{cc}
I & \hat{C}_{f f} \\
\hat{C}_{f f}^{T} & \alpha I
\end{array}\right]>0,} \\
\hat{D}_{f f}+\hat{D}_{f f}^{T}<2 k_{D} I, \\
{\left[\begin{array}{cc}
\mathcal{A}^{T} P_{1}+P_{1} \mathcal{A} & P_{1} \mathcal{B}-\mathcal{C}^{T} \\
\mathcal{B}^{T} P_{1}-\mathcal{C} & -\left(\mathcal{D}+\mathcal{D}^{T}\right)
\end{array}\right]<0,}
\end{gathered}
$$






Figure 6: Offset free control for Hammerstein systems using feedforward passivity design

$$
\begin{gathered}
{\left[\begin{array}{cc}
\mathcal{A}^{T} P_{2}+P_{2} \mathcal{A} & P_{2} \mathcal{B}-\mathcal{C}_{f f}^{T} \\
\mathcal{B}^{T} P_{2}-\mathcal{C}_{f f} & -\left(\mathcal{D}_{f f}+\mathcal{D}_{f f}^{T}\right)
\end{array}\right]<0} \\
P_{1}>0 \\
P_{2}>0 .
\end{gathered}
$$

The LMIs (21) is equivalent to:

$$
\alpha I-\hat{C}_{f f} \hat{C}_{f f}^{T}>0
$$

By minimizing $\alpha$, a lower bound for the maximum singular value of $\hat{C}_{f f}$ as in (21), desired dynamic tracking performance could be achieved.

The dissipativity (described by (19)) of the nonlinearity can be checked numerically (see Section 4). An input transformation can be performed on the static nonlinearity $N L(\cdot)$ such that the transformed function satisfies Condition (19). A special case of such transformation is the inverse of $N L(\cdot)$ if the static nonlinearity is invertible and exactly known (as in [9] and [7]).

The integral controller $C(s)$ given in Theorem 12 can be of any form, e.g., PI, PID controllers. It should be noted that the constraints (23) and (24) of Problem 13 are Bilinear Matrix Inequalities (BMIs) for the decision variables $\hat{C}_{f f}, \hat{D}_{f f}, P_{1}$ and $P_{2}$. Although optimization problems with BMIs constraints could be solved by successive linear programming (SLP) iteratively [20], the global optimum is hard to find because such optimization problems are not convex [30]. However, if $C(s)$ is chosen to have a muti-loop PI form, Problem 13 is reduced to an optimization problem with LMIs

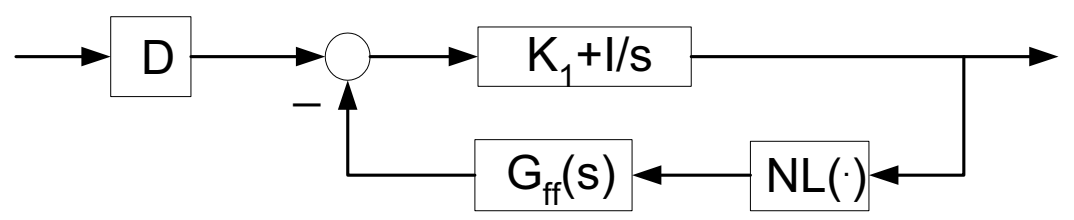

Figure 7: The structure of the PI type controller 
constraints, which can be easily solved by using any SDP solver (e.g., Matlab LMI Toolbox ${ }^{[31]}$ ). As shown in Figures 6 and 7, the PI type controller has the parameters $K_{1}=\operatorname{diag}\left(k_{11}, k_{12}, \cdots, k_{1 m}\right)$ with $k_{1 j}>0 j=1,2, \cdots, m$, and $K_{2}=\operatorname{diag}\left(k_{21}, k_{22} \cdots, k_{2 m}\right)$ with $k_{2 j}>0 j=1,2, \cdots, m$. A real matrix $D^{\prime}$ is designed (which equals $G_{0}^{-1}(0)$ ) to satisfy Condition i) of Theorem 12. Conditions ii), iii) and iv) in Theorem 12 can then be simplified as follows:

ii) $\left(K_{1} s+I\right)\left(\hat{G}(s)+\hat{G}_{f f}(s)\right)+\hat{D}_{f f}+K_{1} K_{2}$ is Positive Real,

iii) $\hat{D}_{f f}+\hat{D}_{f f}^{T}<2 k_{D} I$ and $k_{D}<\rho$,

iv) $\left(K_{1} s+I\right) \hat{G}_{f f}(s)+\hat{D}_{f f}$ is Positive Real.

A design procedure is then presented for the integral control system design:

Procedure 14 The following steps are suggested for parameter selection of our proposed method.

1) Design a single loop PI controller for each diagonal loop of the linear system $G(s)$ using any multi-loop PI control tuning methods, such as the Ziegler-Nichols method. The initial choice of $K_{1}$ and $K_{2}$ are obtained.

2) Select the parameter $k_{D}<\rho$. Use any LMI solver (such as Matlab LMI toolbox) to solve Problem 13. The feedforward system $G_{f f}(s)$ and a minimized value $\alpha$ (see (21)) can be obtained based on the solution.

3) Construct the overall controller (as shown in Figure 7). Perform closed-loop simulation with the controller designed. Observe the dynamic response of the controlled system. If the tracking is not fast enough, increase the value of $k_{D}$ and go back to Step 2). Normally, increasing $k_{D}$ will decrease the minimized value of $\alpha$ which leads to fast tracking performance. However, this is likely to degrade the robustness of the control system.

4) If the desired tracking performance can not be obtained by adjusting $k_{D}$, go back to Step 1) and tune the PI alike parameters $K_{1}$ and $K_{2}$. Repeat steps 2), 3) and 4) until a desired tracking performance is achieved. Normally, increasing the value of $\left(K_{1} K_{2}\right)$ can improve the dynamic tracking speed but may lead to large overshoots in the process response. Increasing $K_{2}$ alone improves the tracking performance near steady state but may also lead to more oscillatory responses.

Robust designs can be achieved using the proposed framework. Assume the static nonlinearity of the Hammerstein model $\hat{N} L(\cdot)$ has a multiplicative model uncertainty, i.e. $N L(\cdot)=\Delta N L(\cdot)+N L(\cdot)$ where $N L(\cdot)$ is the nonlinearity of the true process. Then, the closed loop (a Hammerstein system under the proposed control) can be shown as in Figure 8. Denote $\Delta P=\left(K_{1} s+I\right) \hat{G}_{f f} \Delta$. Recall 


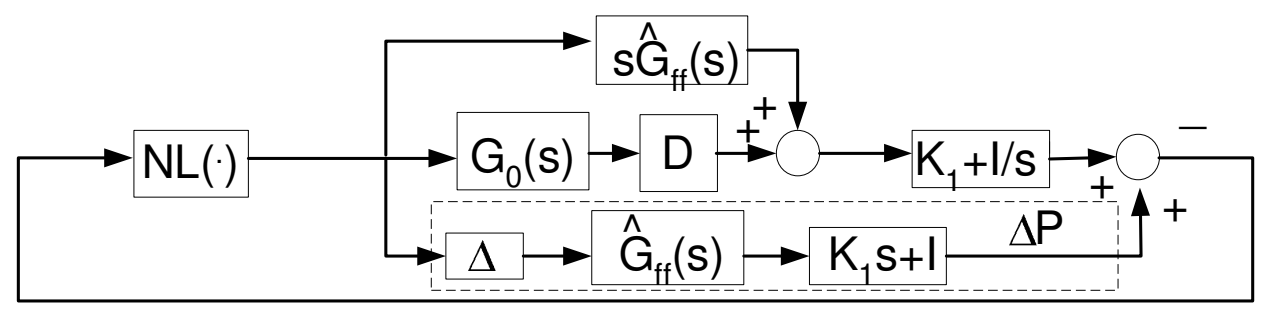

Figure 8: Offset free control for Hammerstein systems with model uncertainty

that the nonlinearity is dissipative with $\nu>0$ and $\rho$, and the designed system $\bar{G}(s)=\frac{K_{1} s+I}{s}[G(s)+$ $\left.s \hat{G}_{f f}(s)\right]$ is $\operatorname{IFP}\left(-k_{D}\right)$. If $\left(\rho-k_{D}\right) I+\Delta P$ is passive (here $\left.\rho-k_{D}>0\right)$, then the controlled system is robustly stable. This robust condition can be satisfied if $\Delta$ is small or $\Delta P$ is $\operatorname{IFP}\left(k_{D}-\rho\right)$. Therefore, $k_{D}$ is a design parameter for robustness. When the parameter $\rho$ is not exactly known, a relatively small $k_{D}$ (which is much less than $\rho$ ) is selected in Procedure 14, according to the estimate of model-plant mismatch. The larger model uncertainty, the smaller $k_{D}$ should be used. Similarly, if there exists uncertainties in the linear dynamics of the Hammerstein model, a more conservative ("over passifying") dynamic feedforward needs to be employed to ensure the linear open loop system with the presence of model uncertainty is passive.

The proposed method guarantees the closed loop stability. It can be designed off-line and implemented easily. Moreover, for the proposed approach, the steady state performance of the system is ensured by the integral control. The dynamic performance is determined by the feedforward subsystem $G_{f f}(s)$. The smaller the $\alpha$, the faster the closed-loop response can be achieved.

\section{Illustrative Example}

In this section, the proposed control method is illustrated using a $\mathrm{pH}$ control example. Existing nonlinear $\mathrm{pH}$ control methods are largely based on input-output linearization ${ }^{[32,33]}$ and nonlinear adaptive control ${ }^{[34,35]}$. If Hammerstein process models are used, these control schemes require the inversion of the static nonlinearity, which is usually difficult and often not possible for multivariable and/or complicated nonlinearities.

Consider an acid-base neutralization process performed in a single tank ${ }^{[36]}$. The system description, the nonlinear process model and the operating conditions can be found in [3]. This process can be described by the following Hammerstein model, where the level $\left(y_{1}\right)$ and $\mathrm{pH}$ of the liquid $\left(y_{2}\right)$ in the well stirred neutralization tank are the controlled variables and the acid and base flow 
rates ( $u_{1}$ and $u_{2}$ respectively) are the manipulated variables.

$$
\begin{gathered}
\dot{x}=\left[\begin{array}{cccc}
-0.1693 & -0.0635 & -0.0552 & 0.0058 \\
-0.1209 & -0.4976 & -0.0901 & 0.0664 \\
4.1895 & 0.6420 & -0.8783 & -0.2147 \\
9.4232 & 7.8142 & -0.0371 & -2.1582
\end{array}\right] x+\left[\begin{array}{cc}
0.1208 & 0.0250 \\
0.1131 & -0.4125 \\
-2.8917 & -1.3531 \\
-6.9287 & 2.7517
\end{array}\right] N L(u) \\
y=\left[\begin{array}{cccc}
1.7389 & -0.4585 & 0.1167 & 0.0053 \\
-0.6846 & -.7915 & -0.0177 & -0.0637
\end{array}\right] x+\left[\begin{array}{cc}
0.0823 & 0.0623 \\
-0.0896 & 0.0402
\end{array}\right] N L(u)
\end{gathered}
$$

and

$N L(u)=\left[\begin{array}{l}u_{1}-0.3056 u_{2}-0.3490 u_{1}^{2}-0.1719 u_{2}^{2}+0.5663 u_{1} u_{2}+0.0322 u_{1}^{3}+0.0326 u_{2}^{3}+0.1987 u_{1} u_{2}^{2}-0.2144 u_{1}^{2} u_{2} \\ 0.0031 u_{1}+u_{2}+0.2896 u_{1}^{2}+0.1983 u_{2}^{2}-0.5149 u_{1} u_{2}-0.0356 u_{1}^{3}+0.0677 u_{2}^{3}-0.2032 u_{1} u_{2}^{2}+0.1452 u_{1}^{2} u_{2}\end{array}\right]$

In the above model, the state $x$, the input $u$ and the output $y$ are all deviation variables, oper-

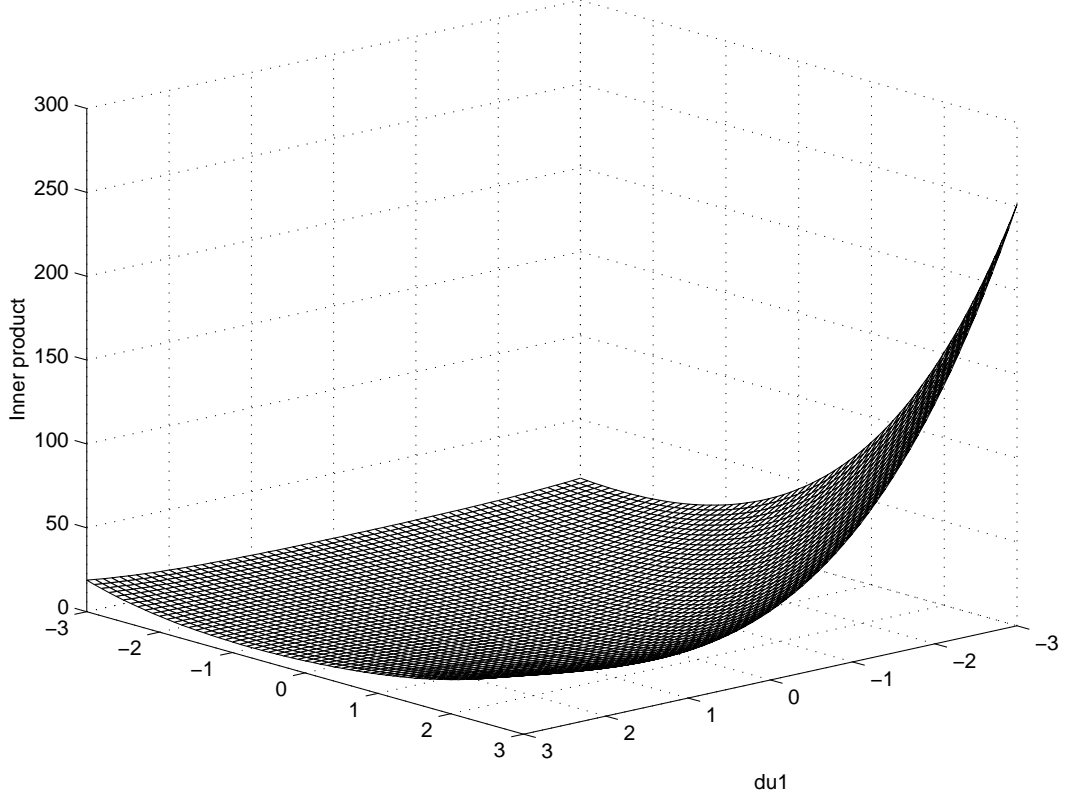

du2

Figure 9: Values of $N L(u)^{T} u$

ating from an equilibrium point where the $\mathrm{pH}$ value of the liquid in the tank is 7 . The reference input in the simulation study is $r=[2,2]^{T}$. The corresponding steady state controller output $(u)$ is $[-0.163,1.573]^{T}$. The dissipativity of the nonlinearity $N L(\cdot)$ needs to be analyzed around this operating point. Because function $N L(u)^{T} u$ is continuous, its dissipativity can be verified numerically using discrete points in the region of interest. A three-dimensional plot is given (around 
$\left.u_{0}=\left[u_{10}, u_{20}\right]^{T}=[-0.163,1.573]^{T}\right)$ in Figure 9. It can be observed numerically that the static nonlinearity is dissipative with $\nu=0.001$ and $\rho=0.05$ around $u_{0}$ in the range of $u_{1} \in\left(u_{10}-3, u_{10}+3\right)$ and $u_{2} \in\left(u_{20}-3, u_{20}+3\right)$. Similarly, the dissipativity of the nonlinearity $N L(\cdot)$ can also be analyzed numerically when there is no reference input. It is verified that the static nonlinearity is dissipative with $\nu=0.01$ and $\rho=0.08$ around $[0,0]^{T}$ in the range of $u_{1} \in(-4,4)$ and $u_{2} \in(-4,4)$. The linear part of this Hammerstein model is non-minimum phase as it has transmission zeros at 0.1426, $0.3532 \pm 1.4549 i$.

Choose $K_{1}=\operatorname{diag}(0.6,0.3), K_{2}=\operatorname{diag}(0.36,0.36)$ and $k_{D}=-0.1$. This implies the GAS of the overall closed loop and the GS of the proposed controller is guaranteed given that the nonlinearity of the Hammerstein model fulfils Condition (19) with $\nu>0$ and $\rho>-0.1$. By using the proposed LMI approach, the designed feedforward subsystem and related parameters are:

$$
\begin{aligned}
& \hat{G}_{f f}=\left[\begin{array}{cc}
\frac{0.1132 s^{3}+1.237 s^{2}+1.557 s+0.1821}{s^{4}+3.703 s^{3}+3.705 s^{2}+1.378 s+0.1494} & \frac{-0.01922 s^{3}-0.1887 s^{2}+0.2064 s-0.09511}{s^{4}+3.703 s^{3}+3.705 s^{2}+1.378 s+0.1494} \\
\frac{0.06816 s^{3}+0.02266 s^{2}+0.02194 s+0.08015}{s^{4}+3.703 s^{3}+3.705 s^{2}+1.378 s+0.1494} & \frac{0.2457 s^{3}+1.021 s^{2}+0.9414 s+0.3122}{s^{4}+3.703 s^{3}+3.705 s^{2}+1.378 s+0.1494}
\end{array}\right] \text {, } \\
& \hat{C}_{f f}=\left[\begin{array}{cccc}
-0.1593 & -0.0857 & -0.0748 & -0.0909 \\
-0.1106 & 0.1811 & -0.0378 & 0.0500
\end{array}\right] \\
& \hat{D}_{f f}=\left[\begin{array}{cc}
-0.0536 & 0.0006 \\
0.0006 & -0.0502
\end{array}\right] \text {, }
\end{aligned}
$$

and $\alpha=0.0490$.

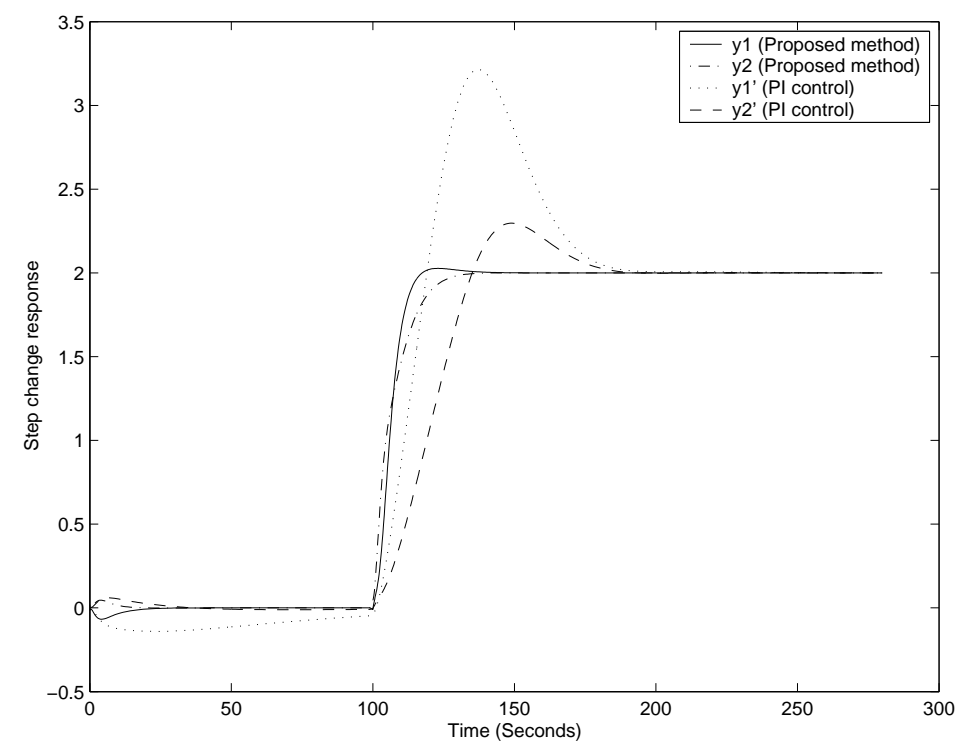

Figure 10: Offset free control for an acid-base neutralization model (output $y$ ) 


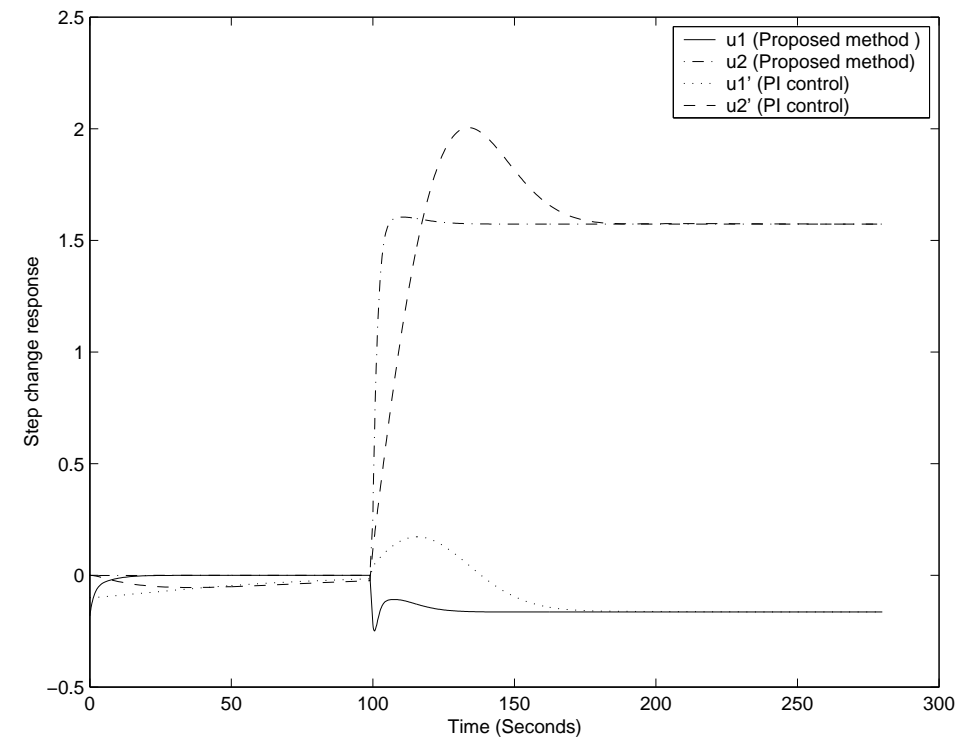

Figure 11: Offset free control for an acid-base neutralization model (input signal $u$ )

The simulation results for the control of the acid-base neutralization Hammerstein model by using the proposed method and a multi-loop PI control method (the parameters of the multi-loop PI controller are tuned by using the "biggest log modulus tuning(BLT) method" ${ }^{[37]}$ ) are compared in Figures 10 and 11. In the simulation study, a constant input disturbance $[0.1,0]^{T}$, which would be the consequence of the unknown concentration changes of the acid flow, is added to the system. The proposed method performs better than multi-loop PI control. The ITAE of output $y_{1}$ for the proposed method is 1453 while that of PI control is 8478 . For output $y_{2}$, the ITAE for the proposed method is 1548 in contrast to 5545 for PI control.

To test the robustness of the proposed control, a simulation study is also performed with model uncertainties in both the static nonlinearity and the linear dynamics. Assume the "true" process is:

$$
\begin{gathered}
\dot{x}=\left[\begin{array}{cccc}
-0.1972 & -0.0817 & -0.0817 & 0.0115 \\
-0.1398 & -0.5124 & -0.1220 & 0.0991 \\
4.2019 & 0.6651 & -0.8957 & -0.2237 \\
9.4466 & 7.8349 & -0.0664 & -2.1666
\end{array}\right] x+\left[\begin{array}{cc}
0.1500 & 0.0548 \\
0.1377 & -0.4191 \\
-2.8962 & -1.3631 \\
-6.9291 & 2.7737
\end{array}\right] N L(u) \\
y=\left[\begin{array}{cccc}
1.7531 & -0.4617 & 0.1458 & 0.0311 \\
-0.7081 & -0.8409 & -0.0389 & -0.0804
\end{array}\right] x+\left[\begin{array}{cc}
0.1039 & 0.0913 \\
-0.1009 & 0.0782
\end{array}\right] N L(u)
\end{gathered}
$$

where,

$$
N L(u)=\left[\begin{array}{c}
u_{1}-0.3036 u_{2}-0.3390 u_{1}^{2}-0.1519 u_{2}^{2}+0.6663 u_{1} u_{2}+0.2987 u_{1} u_{2}^{2}-0.3144 u_{1}^{2} u_{2} \\
0.0021 u_{1}+u_{2}+0.2796 u_{1}^{2}+0.1783 u_{2}^{2}-0.6149 u_{1} u_{2}-0.1356 u_{1}^{3}+0.3677 u_{2}^{3}-0.3032 u_{1} u_{2}^{2}+0.2452 u_{1}^{2} u_{2}
\end{array}\right]
$$


Compared to the process model used in control design, the "true" process has about $5 \%$ variation in each element in the linear part of the Hammerstein model and about $3 \%$ variation in the coefficients of the static nonlinearity. Moreover, the input nonlinearity of the real process does not have the cubic terms of $u_{1}^{3}$ and $u_{2}^{3}$ in the first input channel. As shown in Figures 12 and 13, the multi-loop PI control leads to severely oscillatory response while the proposed control achieves significantly better performance. The proposed method is found to be more robust with model uncertainty of the Hammerstein model.

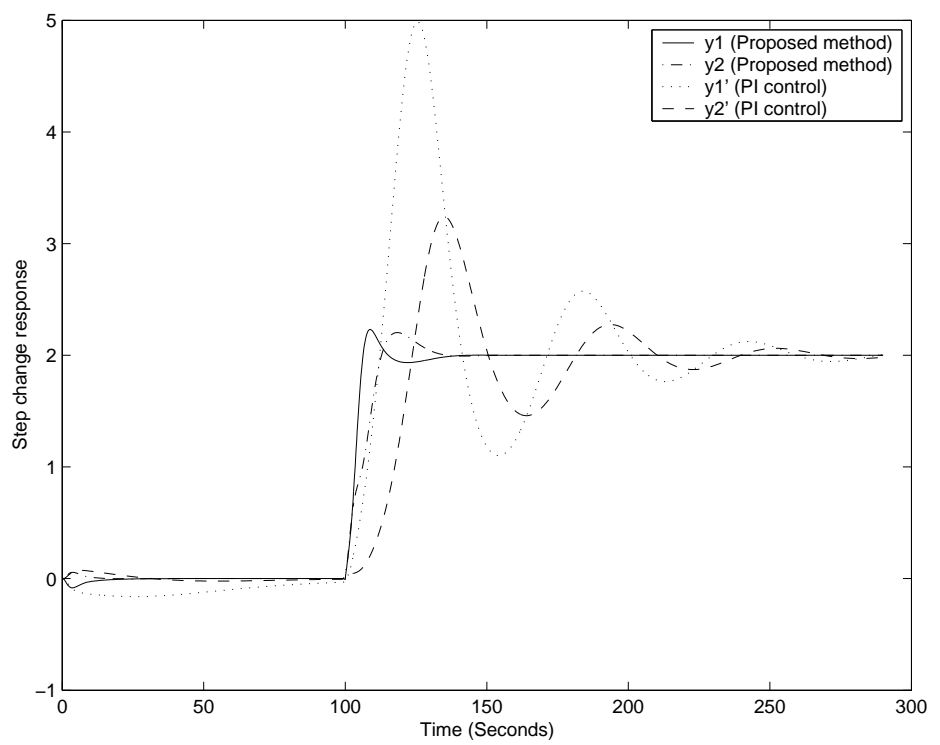

Figure 12: Offset free control for an acid-base neutralization model with model uncertainty (output y)

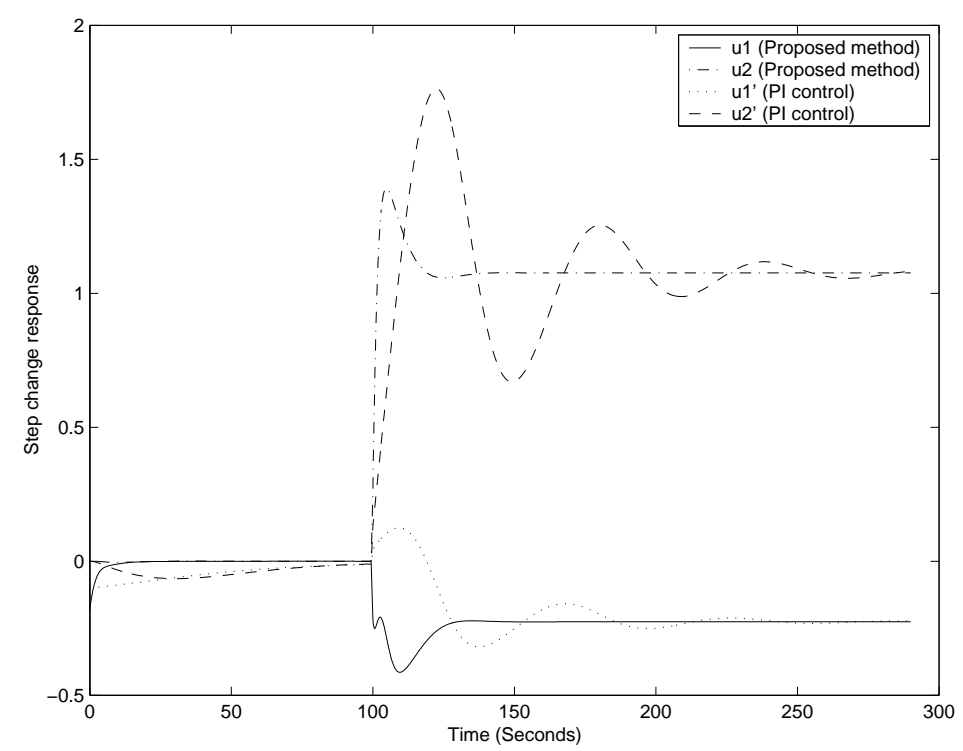

Figure 13: Offset free control for an acid-base neutralization model with model uncertainty (input u) 


\section{Conclusion}

This paper applies integral control to achieve offset free tracking for Hammerstein systems. The stability of the overall closed loop consisting of a linear block with integral action and nonlinearity is obtained by using a feedforward passivation design based on the Passivity Theorem. The construction of the feedforward system is implemented by solving a set of linear matrix inequalities (LMI) derived from the Positive Real Lemma. A stable (GS) offset free controller is designed for a two-input two-output neutralization process (whose linear part is non-minimum phase) based on the proposed method. The proposed approach, in contrast to feedback passivation methods (such as [23]), is not limited to processes with minimum phase and/or relative degree of no more than one. Furthermore, robust stability can be achieved within the same design framework.

\section{Nomenclature}

$\mathcal{R} \quad$ field of real numbers

e $\quad$ field of complex numbers

$\omega \quad$ frequency

$\operatorname{Re}(s) \quad$ real part of $s \in \mathcal{C}$

$A^{T} \quad$ transpose of $A$

$A^{*} \quad$ complex conjugate transpose of $A$

$A>0$ matrix $A$ is positive definite

$N L(\cdot) \quad$ the nonlinearity of a Hammerstein system

\section{Acknowledgments}

The authors gratefully acknowledge the financial support of the Australian Research Council (Grant A00104473).

\section{A Proof of Theorem 9.}

Proof See Figure 4. Because it is assumed that $r=0$, the overall closed loop is unforced. According to the assumption that $(0,0)$ is a Globally Asymptotically Stable equilibrium of the unforced closed 
loop $\left(P, \frac{K_{c}}{s}\right)$, we can conclude that:

$$
\lim _{t \rightarrow \infty} y(t)=0
$$

Then, $u$ should be constant when time goes to infinite. Because $P_{f f}$ has zeros at steady state, we have that:

$$
\lim _{t \rightarrow \infty} y_{f f}(t)=0 .
$$

Then, we conclude that:

$$
\lim _{t \rightarrow \infty} y_{0}(t)=\lim _{t \rightarrow \infty} y(t)-\lim _{t \rightarrow \infty} y_{f f}(t)=0 .
$$

\section{References}

[1] E. Eskinat, S. Johnson, and W. Luyben, "Use of Hammerstein models in identification of nonlinear systems," AIChE Journal, vol. 37, pp. 255-268, 1991.

[2] N. Kapoor, T. McAvoy, and T. Marlin, "Effect of recycle structure on distillation tower time constants," AIChE Journal, vol. 32, p. 41, 1986.

[3] M. Henson and D. Seborg, "Adaptive nonlinear control of a $\mathrm{pH}$ neutralization process," IEEE Transactions on Control Systems Technology, vol. 2, pp. 169-182, 1994.

[4] R. Pearson and M. Pottmann, "Gray-box identification of block-oriented nonlinear models," Journal of Process Control, vol. 10, pp. 301-315, 2000.

[5] B. Ydstie, M. Golden, and S. Chesna, "Adaptive nonlinear model control," Chemical Engineering Communications, vol. 63, pp. 17-37, 1988.

[6] D. Pomerleau, A. Pomerleau, D. Hodouin, and E. Poulini, "A procedure for the design and evaluation of decentralised and model-based predictive multivariable controllers for a pellet cooling process," Computers 63 Chemical Engineering, vol. 27, pp. 217-233, 2003.

[7] C. Hwang and J. Hsu, "Nonlinear control design for Hammerstein model system," IEE Proc.Control Theory Applications, vol. 142, pp. 277-285, 1995. 
[8] K. Fruzzetti, A. Palazoglu, and K. McDonald, "Nonlinear model predictive control using Hammerstein models," Journal of Process Control, vol. 7, pp. 31-41, 1997.

[9] F. M'sahli, R. Abdennour, and M. Ksouri, "Nonlinear model-based predictive control using a generalised Hammerstein model and its application to a semi-batch reactor," Int. J. Adv. Manuf. Technol., vol. 20, pp. 844-852, 2002.

[10] J. Ibarrola, J. Sandoval, M. Garca-Sanz, and M. Pinzolas, "Predictive control of a high temperature-short time pasteurisation process," Control Engineering Practice, vol. 10, pp. 713$725,2002$.

[11] W. Chen, D. Ballance, and P. Gawthrop, "Optimal control of nonlinear systems: a predictive control approach," Automatica, vol. 39, no. 4, pp. 633-641, 2003.

[12] M. Morari and J. Leeb, "Model predictive control: past, present and future," Computers $\&$ Chemical Engineering, vol. 23, pp. 667-682, 1999.

[13] Z. Jiang and I. Mareels, "Robust nonlinear integral control by partial-state and output feedback," Proceedings of the IEEE Conference on Decision and Control, pp. 2084-2089, 2000.

[14] M. Morari and L. Zafiriou, Robust Process Control. Englewood Cliffs: Prentice-Hall, 1989.

[15] D. Rivera, M. Morari, and S. Skogestad, "Internal model control, 4. PID controller design," Ind. Eng. Process Design Dev., vol. 25, pp. 252-265, 1986.

[16] O. Smith, "Closer control of loops with dead time," Chem. Eng. Prog., vol. 53, pp. 217-219, 1957.

[17] E. Mishkin and L. Braun, Adaptive Control Systems. New York: the McGraw-Hill, 1961.

[18] H. Kaufman, I. Bar-Kana, and K. Sobel, Direct Adaptive Control Algorithms: Theory and Applications. New York: Springer-Verlag, 1994.

[19] R. Sepulchre, M. Jankovic, and P. Kokotovic, Constructive Nonlinear Control. New York: Springer Verlag, 1996.

[20] J. Bao, W. Zhang, and P. Lee, "Decentralized fault-tolerant control system design for unstable processes," Chemical Engineering Science, vol. 58, pp. 5045-5054, 2003. 
[21] D. Bernstein and W. Haddad, "Nonlinear controllers for positive real systems with arbitrary input nonlinearities," IEEE Transactions on Automatic Control, vol. 39, no. 7, pp. 1513-1517, 1994.

[22] H. Sane and D. Bernstein, "Asymptotic disturbance rejection for hammerstein positive real systems," IEEE Transactions on Control Systems Technology, vol. 11, no. 3, pp. 364-374, 2003.

[23] J. Bernussou, J. Geromel, and M. de Oliveira, "On strict positive real systems design: Guaranteed cost and robustness issues," Systems and Control Letters, vol. 36, pp. 135-141, 1999.

[24] A. van der Schaft, $\mathcal{L}_{2}$ Gain and Passivity Techniques in Nonlinear Control. New York: Springer Verlag, 1996.

[25] H. Sira-Ramirez and M. Angulo-Nunez, "Passivity-based control of nonlinear chemical processes," International Journal of Control, vol. 68, no. 5, pp. 591-996, 1997.

[26] J. Willems, "Dissipative dynamical systems, parts I and II," Arch. Ration. Mech. Anal., vol. 45, pp. 321-393, 1972.

[27] W. Sun, P. Khargonekar, and D. Shim, "Solution to the positive real control problem for linear time-invariant systems," IEEE Transactions on Automatic Control, vol. 39, no. 10, pp. 20342046, 1994.

[28] S. Su, B. Anderson, and T. Brinsmead, "Use of integrator in nonlinear $\mathcal{H}_{\infty}$ design for disturbance rejection," Automatica, vol. 38, no. 11, pp. 1951-1957, 2002.

[29] S.M.Joshi and S.Guta, "On a class of marginally stable positive-real systems," IEEE Transactions on Automatic Control, vol. 41, no. 1, pp. 152-155, 1996.

[30] K. Goh, M. Safonov, and J. Ly, "Robust synthesis via bilinear matrix inequalities," International Journal of Robust Nonlinear Control, vol. 6, pp. 1079-1095, 1996.

[31] P. Gahinet, A. Nemirovski, A. Laub, and M. Chilali, LMI Control Toolbox: For use with MATLAB. The MathWorks, Inc., 1995.

[32] T. Gustafsson and K. Waller, "Dynamic modeling and reaction invariant control of pH," Chemical Engineering Science, vol. 38, pp. 289-398, 1983. 
[33] K. Waller and P. Makila, "Chemical reaction invariants and variants and their use in reactor modeling, simulation, and control," Ind. Eng. Chem. Proc. Design and Develop, vol. 20, pp. 1$11,1981$.

[34] M. Henson and D. Seborg, "Adaptive nonlinear control of a $\mathrm{pH}$ neutralization process," IEEE Transactions Control Systems Technology, vol. 2, pp. 169-182, 1994.

[35] T. Gustafsson and K. Waller, "Nonlinear and adaptive control of pH," Industrial \&3 Engineering Chemistry Research, vol. 31, pp. 2681-2693, 1992.

[36] S. Lakshminarayanan, S. Shah, and K. Nandakumar, "Identification of Hammerstein models using multivariable statistical tools," Chemical Engineering Science, vol. 50, pp. 3599-3613, 1995.

[37] W. Luyben, "Simple method for tuning SISO controllers in multivariable systems," Ind. Eng. Chem. Process Des. Dev., vol. 25, pp. 654-660, 1986. 